Pacific Journal of Mathematics

HERMITIAN AND ADJOINT ABELIAN OPERATORS ON 


\title{
HERMITIAN AND ADJOINT ABELIAN OPERATORS ON CERTAIN BANACH SPACES
}

\author{
Richard J. Fleming AND JAMES E. JAMISON
}

\begin{abstract}
Let $X$ be a complex linear space endowed with a semiinner product $[\cdot, \cdot]$. An operator $A$ on $X$ will be called Hermitian if $[A x, x]$ is real for all $x \in X ; A$ is said to be adjoint abelian if $[A x, y]=[x, A y]$ for all $x$ and $y \in X$. Since every Banach space may be given a semi-inner product (not necessarily unique) which is compatible with the norm, it is possible to study such operators on general Banach spaces. This paper characterizes Hermitian and adjoint abelian operators on certain Banach spaces which decompose as a direct sum of Hilbert spaces. In particular, the Hermitian operators are shown to have operator matrix representations which are diagonal, with the operators on the diagonal being Hermitian operators on the appropriate Hilbert space. The class of spaces studied includes those Banach spaces with hyperorthogonal Schauder bases.
\end{abstract}

The notion of semi-inner product is due to Lumer [4] who also introduced the concept of numerical range of an operator on a Banach space in terms of a semi-inner product. This led to the definition of Hermitian operator given above as an operator with real numerical range. Another approach to the idea of numerical range appeared about the same time in the work of Bauer [1]. A good account of developments in the theory of Banach space numerical ranges is given in [3].

In connection with this work on isometries of reflexive Orlicz spaces, Lumer [5] has shown that the Hermitian operators on a reflexive Orlicz space over a nonatomic measure space are essentially multiplications by real $L^{\infty}$-functions. Tam [10] obtained this same result for Hermitian operators on discrete, symmetric Banach function spaces with absolutely continuous norm.

A recent paper of Schneider and Turner [7], making use of Bauer's approach to numerical ranges, characterizes Hermitian matrices (on finite dimensional spaces with absolute norm) in terms of a certain equivalence relation on the coordinates. The results of that paper have motivated the present work. The property of a norm being absolute can be expressed as a property of the basis; indeed to say that a norm is absolute in the sense used in [7] is to say that the natural unit vector basis is hyperorthogonal. By utilizing the notion of hyperorthogonal Schauder basis we are able to extend the results of [7] on Hermitian operators to Banach spaces with this type of basis. 
Our methods are slightly different in that we make use of an explicit representation of the semi-inner product. Our results also extend Tam's theorem [10, Theorem 2] by removing the symmetry condition.

In $\S 2$ we consider a class of Banach spaces which have what we call Hilbert-decompositions and which have semi-inner products with certain nice properties. We then characterize Hermitian operators on these spaces [Theorem 2.6] and obtain some corollaries concerning normal operators and products of Hermitian operators. In $\S 4$ we show that the class includes Banach spaces with hyperothogonal Schauder bases, thus obtaining the results mentioned in the preceding paragraph.

Stampfli [9] introduced the concept of adjoint abelian operator on a Banach space. Our definition depends on the particular choice of semi-inner product and therefore agrees with Stampfli's definition on smooth Banach spaces. In $\S 3$, we characterize these operators on Banach spaces which are $l_{p}$-sums of Hilbert spaces $1<p<\infty$, $(p \neq 2)$. The class of adjoint abelian operators is generally distinct from the class of Hermitian operators, although in some cases it may be a proper subclass. We do show that if a Banach space $X$ is isomorphic to Hilbert space then the classes of adjoint abelian and Hermitian operators coincide if and only if $X$ is isometrically isomorphic to Hilbert space. In particular, the two classes are always distinct on finite dimensional non-Euclidean spaces.

\section{Hermitian operators.}

Definition 2.1. A semi-inner product (s.i.p.) on a linear space $X$ is a mapping $[\cdot, \cdot]$ from $X \times X$ into the scalar field which satisfies

$$
\begin{gathered}
{[x+y, z]=[x, z]+[y, z] \text { for all } x, y, z \in X,} \\
{[\lambda x, y]=\lambda[x, y] \text { for } \lambda \text { a scalar },} \\
{[x, x]>0 \text { for } x \neq 0 .} \\
|[x, y]|^{2} \leqq[x, x][y, y] \text { for all } x, y \in X .
\end{gathered}
$$

Lumer has shown that if $(X, \nu)$ is a Banach space and $\phi$ is a duality map from $X$ into $X^{*}$ with dual norm $\nu^{*}$ such that $\nu^{*}(\phi x)=\nu(x)$, $\phi x(x)=[\nu(x)]^{2}$, then $[x, y]=\phi y(x)$ defines a s.i.p. on $X$ which is compatible with the norm; i.e., $\nu(x)=[x, x]^{1 / 2}$. Such duality maps always exist but are generally not unique unless the space is smooth [4]. In addition one can assume that $\phi \lambda x=\bar{\lambda} \phi x$ for each $x$.

Definition 2.2. Let $(X,[\cdot, \cdot])$ be a complex semi-inner product space. If $A$ is an operator on $X$ then the numerical range $W(A)$ of 
$A$ is defined by $W(A)=\{[A x, x]:[x, x]=1\}$.

If $X$ is an inner product space then it is well known that $A$ is self adjoint or Hermitian if and only if $W(A)$ is real. Lumer [4] has shown that if $X$ is a Banach space and $A$ is an operator on $X$ then the numerical ranges of $A$ relative to s.i.p.'s which are compatible with the norm must have the same convex hull. Thus if $W(A)$ is real for a given s.i.p. which is compatible with the norm, then $W(A)$ is real for every such s.i.p. This makes the next definition possible.

Definition 2.3. An operator $A$ on a Banach space $X$ is said to be Hermitian if $[A x, x]$ is real for every $x$, where $[\cdot, \cdot]$ is a s.i.p. compatible with the norm.

Our purpose in this section is to characterize Hermitian operators on Banach spaces which are direct sums of Hilbert spaces and which possess s.i.p.'s which can be represented in a natural way.

Definition 2.4. A Banach sequence space $(E, \mu)$ has an absolute norm if given $\left\{x_{i}\right\} \in E$ and $\left\{y_{i}\right\}$ with $\left|y_{i}\right|=\left|x_{i}\right|$ for each $i$ then $\left\{y_{i}\right\} \in E$ and $\mu\left(\left\{y_{i}\right\}\right)=\mu\left(\left\{x_{i}\right\}\right)$. A Banach space $(X, \nu)$ is said to have an $H$-decomposition if there exists a sequence of subspaces $\left\{X_{i}\right\}$, each a Hilbert space, and a Banach sequence space $(E, \mu)$ with absolute norm such that (i) the $X_{i}$ 's form a decomposition of $X$, i.e., every $x \in X$ can be represented uniquely as $x=\sum x_{i}, x_{i} \in X_{i}$, (ii) if $x=\sum x_{i}$, then $\left\{\left\|x_{i}\right\|\right\} \in E$ and $\nu(x)=\mu\left(\left\{\left\|x_{i}\right\|\right\}\right)$ and (iii) $\mu\left(\left\{u_{k}\right\}\right)=1$ for any sequence of the form $u_{k}=1$ for $k=i$ and $u_{k}=0$ for $k \neq i$.

REMARKS. If a Banach sequence space $(E, \mu)$ has an absolute norm, then if $\left\{x_{i}\right\},\left\{y_{i}\right\} \in E$ and $\left|y_{i}\right| \leqq\left|x_{i}\right|$ it follows that $\mu\left(\left\{y_{i}\right\}\right) \leqq$ $\mu\left(\left\{x_{i}\right\}\right)$. An example of a Banach space with an $H$-decomposition is an $l_{p}(\infty>p \geqq 1)$ sum of a sequence $\left\{X_{i}\right\}$ of Hilbert spaces. Other examples will be given in $\S 4$.

Definition 2.5. Let $(X, \nu)$ be a Banach space possessing an $H$-decomposition $\left(\left\{X_{i}\right\},(E, \mu)\right)$. A semi-inner product $[\cdot, \cdot]$ on $X$ will be called "sufficiently $l_{p}$-like" if there exists a sequence of nonnegative functions $\left\{a_{i}\right\}$ defined on $X$ with the properties: For $x=\sum x_{i}, y=\sum y_{i}$.

(2.5.1) $a_{k}(x)=a_{k}(y)$ for each $k$ if $\left\|x_{i}\right\|=\left\|y_{\imath}\right\|$ for every $i$,

(2.5.2) $a_{\imath}(\lambda x)=a_{i}(x)$ for every nonzero scalar $\lambda$,

(2.5.3) for every pair $k \neq j$ there exist $x_{k} \in X_{k}, x_{j} \in X_{j}$ such that for $x=x_{k}+x_{j}, a_{k}(x)=a_{j}(x) \neq 0$,

(2.5.4) for every pair $k \neq j$ there exists $x \in X$ with $x_{k} \neq 0, x_{\jmath} \neq 0$ such that $a_{k}(x) \neq a_{j}(x)$, 
(2.5.5) $[x, y]=\Sigma\left\langle x_{i}, y_{i}\right\rangle a_{i}(y)$ where $\langle\cdot, \cdot\rangle$ denotes the s.i.p. on $X_{i}$.

REMARK. If $X$ is an $l_{p}$-sum of Hilbert spaces $\left\{X_{i}\right\}$ where $1<$ $p<\infty(p \neq 2)$, then an example of a semi-inner product which is sufficiently $l_{p}$-like in the sense of 2.5 is given by: $[x, y]=\sum\left\langle x_{i}, y_{i}\right\rangle a_{i}(y)$, where

$$
a_{\imath}(y)=\left(\frac{\left\|y_{i}\right\|}{\|y\|}\right)^{p-2} \text { for }\|y\| \neq 0 .
$$

In fact in this case the s.i.p. is unique.

If $(X, \nu)$ is a Banach space which has an $H$-decomposition $\left(\left\{x_{i}\right\}\right.$, $(E, \mu)$ ) then every operator on $X$ can be represented by an operator matrix. For each positive integer $k$ define the projection operator $P_{k}$ by $P_{k} x=x_{k}$. Then for each pair of positive integers $i, j$ define the operator $A_{i j}$ from $X_{j}$ to $X_{i}$ by $A_{i j}=P_{i} A_{j}$ where $A_{j}$ is the restriction of $A$ to $X_{j}$. Now $A$ can be represented by the operator matrix

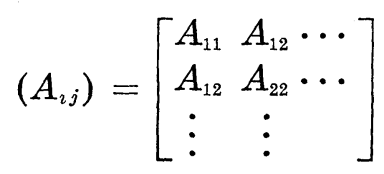

whose action on a vector $x=\sum_{i} x_{i}$ is interpreted in the obvious way. In the remainder of the paper, we will say a Banach space belongs to the class $\mathscr{S}$ if it has an $H$-decomposition and possesses a sufficiently $l_{p}$-like semi-inner product.

THEOREM 2.6. Let $(X, \nu)$ be a Banach space which possesses an $H$-decomposition and suppose $(X, \nu)$ possesses a sufficiently $l_{p}$-like s.i.p. An operator $A$ on $X$ with operator matrix $\left(A_{i j}\right)$ is Hermitian if and only if $\left(A_{i i}\right)$ is Hermitian for each $i$ and $A_{i j}=0$ for $i \neq j$. Furthermore, $\nu_{0}(A)=\operatorname{Sup}_{i}\left\|A_{i i}\right\|$ where $\nu_{0}$ denotes the operator norm.

Proof. Let $\left\{X_{i}\right\}$ denote the family of Hilbert spaces which decompose $X$ and let $\langle\cdot, \cdot\rangle,\|\cdot\|$ denote the inner product and the associated norm respectively on $X_{i}$.

First suppose $A$ satisfies the condition given in the statement of the theorem. If $x=\sum_{i} x_{i} \in X$ then $[A x, x]=\sum_{i}\left\langle A_{i i} x_{i}, x_{i}\right\rangle a_{i}(x)$ where the $a_{i}$ 's satisfy the conditions given in 2.5. Since $A_{k k}$ is Hermitian for each $k$ and the $a_{i}$ 's are real, $[A x, x]$ is real. Thus $A$ is Hermitian.

Suppose now that $A$ is Hermitian. Let $i$ be given and $x_{i}$ an arbitrary nonzero element of $X_{i}$. Then

$$
\left[A x_{i}, x_{i}\right]=\left\langle A_{i i} x_{i}, x_{i}\right\rangle a_{i}\left(x_{i}\right)=\left\langle A_{i i} x_{i}, x_{i}\right\rangle
$$

must be real. It follows that $A_{i i}$ is Hermitian as an operator on $X_{i}$. 
Now let $k, j$ be given with $k \neq j$ and $x_{k}, x_{j}$ be arbitrary elements of $X_{k}$ and $X_{j}$.respectively. Let $x=x_{k}+x_{j}$. Then

$$
\begin{aligned}
{[A x, x]=} & \left\langle A_{k k} x_{k}, x_{k}\right\rangle a_{k}(x)+\left\langle A_{k j} x_{j}, x_{k}\right\rangle a_{k}(x) \\
& +\left\langle A_{j k} x_{k}, x_{j}\right\rangle a_{j}(x)+\left\langle A_{j j} x_{j}, x_{j}\right\rangle a_{j}(x) .
\end{aligned}
$$

The first and last terms have already been shown to be real. Since $[A x, x]$ is real we have for all choices of $x_{k} \in X_{k}$ and $x_{j} \in X_{j}$, (2.6.1) $\left\langle A_{k j} x_{j}, x_{k}\right\rangle a_{k}(x)+\left\langle A_{j k} x_{k}, x_{j}\right\rangle a_{j}(x)$ is real. We proceed to show that $A_{k j}=0$ and $A_{j k}=0$. From (2.6.1) we get

$$
\begin{aligned}
& \left\langle A_{k j} x_{j}, x_{k}\right\rangle a_{k}(x)+\left\langle A_{j k} x_{k}, x_{j}\right\rangle a_{j}(x) \\
= & \left\langle\overline{A_{k j} x_{j}, x_{k}}\right\rangle a_{k}(x)+\left\langle\overline{A_{j k} x_{k}, x_{j}}\right\rangle a_{j}(x) .
\end{aligned}
$$

If we let $x^{\prime}=i x_{k}+x_{j}$, then $a_{k}\left(x^{\prime}\right)=a_{k}(x)$ and $a_{j}\left(x^{\prime}\right)=a_{j}(x)$ by (2.5.1). The substitution of $x^{\prime}$ into (2.6.2) yields

$$
\begin{aligned}
& -i\left\langle A_{k j} x_{j}, x_{k}\right\rangle a_{k}(x)+i\left\langle A_{j k} x_{k}, x_{j}\right\rangle a_{j}(x) \\
= & i\left\langle\overline{\left\langle A_{k j} x_{j}, x_{k}\right.}\right\rangle a_{k}(x)-i\left\langle\bar{A}_{j k} x_{k}, x_{j}\right\rangle a_{j}(x) .
\end{aligned}
$$

Dividing through (2.6.3) by $i$ and adding to (2.6.2) leads to

(2.6.4) $\left\langle A_{j_{k}} x_{k}, x_{j}\right\rangle a_{j}(x)=\left\langle\overline{A_{k j} x_{j}, x_{k}}\right\rangle a_{k}(x)$ which holds for all choices of $x_{k} \in X_{k}$ and $x_{j} \in X_{j}$.

It follows from (2.5.3) that there exists $x_{k}^{\prime} \in X_{k}$ and $x_{j}^{\prime} \in X_{j}$ such that $a_{k}\left(x^{\prime}\right)=a_{j}\left(x^{\prime}\right) \neq 0$ for $x=x_{k}^{\prime}+x_{j}^{\prime}$. If $x_{k}, x_{j}$ are arbitrary nonzero elements of $X_{k}$ and $X_{j}$ respectively and $x=\left\|x_{k}^{\prime}\right\|\left(x_{k} /\left\|x_{k}\right\|\right)+$ $\left\|x_{j}^{\prime}\right\|\left(x_{j} /\left\|x_{j}\right\|\right)$ it follows from (2.5.1) that $a_{k}(x)=a_{j}(x) \neq 0$ and from (2.6.4) we have

$$
\left\langle A_{k j} x_{j}, x_{k}\right\rangle=\left\langle\overline{A_{j k} x_{k}, x_{j}}\right\rangle .
$$

Combining (2.6.5) and (2.6.1) we see that

(2.6.6) $\left.\left\langle A_{k j} x_{j}, x_{k}\right\rangle a_{k}(x)+\overline{\left\langle A_{k j} x_{j}, x_{k}\right.}\right\rangle a_{j}(x)$ is real for all choices of $x_{k} \in X_{k}$ and $x_{j} \in X_{j}$.

It is clear that (2.6.6) will be contradicted if there exists $x_{k} \in X_{k}$, $x_{j} \in X_{j}$ with $\left\langle A_{k j} x_{j}, x_{k}\right\rangle \neq 0$ and $a_{k}(x) \neq a_{j}(x)$ where $x=x_{k}+x_{j}$.

Suppose then that $a_{k}(x)=a_{j}(x)$ for every $x=x_{k}+x_{j}$ such that $\left\langle A_{k j} x_{j}, x_{k}\right\rangle \neq 0$. If $A_{k j} \neq 0$, then there exists $x_{k} \in X_{k}$ and $x_{j} \in X_{j}$ such that $\left\langle A_{k j} x_{j}, x_{k}\right\rangle \neq 0$. If $y=y_{k}+y_{j}$ is given, then $\left\langle A_{k j} z_{j}, z_{k}\right\rangle \neq 0$ where $z_{j}=\left\|y_{j}\right\|\left(x_{j} /\left\|x_{j}\right\|\right), z_{k}=\left\|y_{k}\right\|\left(x_{k} /\left\|x_{k}\right\|\right)$. By (2.5.1) and our assumption, $\boldsymbol{a}_{k}(y)=a_{j}(y)$.

Hence, in order that (2.6.6) fail to be contradicted if $A_{k j} \neq 0$, we must have $a_{k}(x)=a_{j}(x)$ for all $x=x_{k}+x_{j}$.

More generally, from (2.6.5) we have 


$$
[A x, x]=\sum_{1 \leqq p<q}\left[\left\langle A_{p q} x_{q}, x_{p}\right\rangle a_{p}(x)+\left\langle\overline{A_{p q} x_{q}, x_{p}}\right\rangle a_{q}(x)\right]
$$

is real for all $x=\sum x_{i} \in X$. For a given $j$, we may group the terms involving $j$ and replace $x$ by $x^{\prime}=x_{1}+x_{2}+\cdots+e^{i \theta} x_{j}+\cdots$ to obtain

$$
\begin{aligned}
& e^{i \theta}\left(\sum_{1 \leqq p<j}\left\langle A_{p j} x_{j}, x_{p}\right\rangle a_{p}(x)+\sum_{q>j}\left\langle\overline{A_{j q} x_{q}, x_{j}}\right\rangle a_{q}(x)\right) \\
& \left.\quad+e^{-i \theta}\left(\sum_{1 \leqq p<j} \overline{\left\langle A_{p j} x_{j}, x_{p}\right.}\right\rangle a_{j}(x)+\sum_{q>j}\left\langle A_{j q} x_{q}, x_{j}\right\rangle a_{j}(x)\right)+\gamma
\end{aligned}
$$

is real for all choices of $\theta, 0 \leqq \theta<2 \pi$ where $\gamma$ is independent of $\theta$. From the lemma of Tam [9, p. 236], and after some straightforward computation, we may conclude that

$$
\begin{aligned}
& \sum_{1 \leqq p<j}\left\langle A_{p j} x_{j}, x_{p}\right\rangle\left(a_{p}(x)-a_{j}(x)\right)+\sum_{q>j}\left\langle\overline{A_{j q} x_{q}, x_{j}}\right\rangle \\
& \quad \times\left(a_{q}(x)-a_{j}(x)\right)=0
\end{aligned}
$$

for any $x$ with $x_{j} \neq 0$. By (2.5.1) we may replace each term in (2.6.8) by its absolute value, and can therefore conclude that $\left\langle A_{k j} x_{j}, x_{k}\right\rangle a_{k}(x)=$ $\left\langle A_{k j} x_{j}, x_{k}\right) a_{j}(x)$ for any choice of $x$. If $\left\langle A_{k j} x_{j}, x_{k}\right\rangle \neq 0$ we conclude that $a_{k}(x)=a_{j}(x)$. It follows that $a_{k}(x)=a_{j}(x)$ for all $x$ if $A_{k j} \neq 0$. But from (2.5.4) we know that $a_{k}(x) \neq a_{j}(x)$ for some $x$ and consequently $A_{k j}=0$. It now follows from (2.6.5) that $A_{j k}$ is also zero.

To complete the proof, we must show that $\nu_{0}(A)=\sup _{i}\left\|A_{i i}\right\|$. Let $M=\sup _{i}\left\|A_{i i}\right\|$. Then for any $x$,

$$
\nu(A x)=\mu\left(\left\{\left\|A_{i i} x_{i}\right\|\right\}\right) \leqq \mu\left(\left\{\left\|A_{i i}\right\| \cdot\left\|x_{i}\right\|\right\}\right) \leqq M \mu\left(\left\{\left\|x_{i}\right\|\right\}\right)=M \nu(x) .
$$

Hence $\nu_{0}(A) \leqq M$. Since it is obvious that $\nu_{0}(A) \geqq M$, we obtain equality and the proof is complete.

In each of the following corollaries, $X$ will denote a Banach space which satisfies the hypothesis of Theorem 2.6.

Corollary 2.7. Let $A, B$ be Hermitian operators on $X$. Then $A B$ is Hermitian if and only if $A B=B A$.

Proof. This follows from the characterization of $A$ and $B$ as having diagonal operator matrix representations and from the corresponding result for Hilbert spaces.

The conclusion of Corollary 2.7 does not hold in general [2, p. 106].

CoRollary 2.8. If $A$ is a Hermitian operator on $X$, then $A^{n}$ is Hermitian for every positive integer $n$. p. 84]. 
Following Lumer [5, p. 79], we say an operator $T$ on a Banach space $X$ is normal if $T=A+i B$ where $A$ and $B$ are Hermitian and $A B=B A$. The next corollary characterizes normal operators in our particular class of spaces.

CoRollary 2.9. Let $T$ be an operator on $X$. Then $T$ is normal if and only if $T$ is represented by an operator matrix $T=\left(T_{k j}\right)$ such that $T_{k j}=0$ for $k \neq j$, and for each $k . T_{k k}$ is normal on an operator on the Hilbert space $X_{k}$.

Proof. It is well known that an operator $K$ on a Hilbert space is normal if and only if $K=R+i J$ where $R, J$ are Hermitian and $R J=J R$. The desired result follows readily from the characterization of Hermitian operators on $X$.

An operator $S$ on a Banach space $(X, \nu)$ is called a scalar operator if there exists a spectral measure $E(\cdot)$ such that $S=\int z d E(z)$.

COROLlaRY 2.10. If $X$ is reflexive then every normal operator is a scalar operator.

Proof. This follows from a theorem of Berkson [6, p. 79] and Corollaries 2.7 and 2.9 .

3. Adjoint abelian operators. Stampfli [9] defines an operator on a Banach space to be adjoint abelian if there is a s.i.p. compatible with the norm such that $[A x, y]=[x, A y]$ for every $x, y$ in the space.

Definition 3.1. An operator $A$ on a s.i.p.s $(X,[\cdot, \cdot])$ is said to be adjoint abelian if and only if $[A x, y]=[x, A y]$ for all $x, y \in X$.

The above definition differs from that of Stampfli in that it is defined relative to a given s.i.p. The following example shows, in contrast to the situation for Hermitian operators, that an operator may be adjoint abelian with respect to one s.i.p. but not with respect to another, even though both are compatible with the norm. This ambiguity does not occur in the case of a smooth Banach space which has a unique s.i.p. compatible with the given norm.

EXAMPLE 3.1.1. Let $X$ be a two dimensional complex space with the $l^{\infty}$ norm. Let $\lambda$ be chosen with $0<\lambda<1$. Let $\phi_{\lambda}$. denote the map defined on $X$ into $X^{*}$ by

$$
\phi_{\lambda}(y)=\left\{\begin{array}{l}
\left(\bar{y}_{1}, 0\right) \text { if }\left|y_{1}\right|>\left|y_{2}\right| \\
\left(0, \bar{y}_{2}\right) \text { if }\left|y_{1}\right|<\left|y_{2}\right| \\
\left(\lambda \bar{y}_{1},(1-\lambda) \bar{y}_{2}\right) \text { if }\left|y_{1}\right|=y_{2} \mid .
\end{array}\right.
$$


It is easy to verify that $\left\|\phi_{\lambda}(y)\right\|_{1}=\|y\|_{\infty}$ and $\phi_{\lambda} y(y)=\|y\|_{\infty}^{2}$. Hence we may define, for each $\lambda$, a s.i.p. on $X$ by $[x, y]_{\lambda}=\phi_{\lambda} y(x)$ such that $[x, y]_{\lambda}$ is compatible with $\|\cdot\|_{\infty}$. Let $A=\left[\begin{array}{ll}0 & 1 \\ 1 & 0\end{array}\right]$. It is clear that $[A x, y]_{\lambda}=[x, A y]_{\lambda}$ for all choices of $x, y$ if and only if $\lambda=1 / 2$. Hence $A$ is adjoint abelian relative to $[\cdot, \cdot]_{\lambda}$ for $\lambda=1 / 2$ but not adjoint abelian relative to $[\cdot, \cdot]_{2}$ for $\lambda \neq 1 / 2$.

We now wish to characterize adjoint abelian operators in a manner similar to that for Hermitian operators. We shall assume here that the Banach space is an $l_{p}$-sum of separable Hilbert spaces, where $1<p<\infty, p \neq 2$ and the Hilbert spaces may be of varying dimension finite or infinite.

In this case, the semi-inner product is given by

$$
[x, y]=\sum\left\langle x_{i}, y_{i}\right\rangle \frac{\left\|y_{i}\right\|^{p-2}}{[\nu(y)]^{p-2}}
$$

where $x=\sum x_{i}, y=\sum y_{i}$ and $\nu$ is the norm on $X$.

TheOREM 3.2. Let $X$ be an $l_{p}$-sum of Hilbert spaces $X_{i}$ where $1<p<\infty, p \neq 2$, with norm $\nu$ and semi-inner product given by (3.1.2). An operator $A \neq 0$ on $X$ with operator matrix representation $\left(A_{k j}\right)$ is adjoint abelian if and only if the following conditions are satisfied:

(3.2.1) $A_{k j}=0$ if $X_{k}$ and $X_{j}$ have different dimension.

(3.2.2) For each $k$, there exists one and only one $j$ such that $A_{k j} \neq 0$.

(3.2.3) If $A_{k j} \neq 0$, then $A_{k j}=A_{j k}^{*}$.

(3.2.4) There is a constant $\lambda>0$ such that if $A_{k j} \neq 0$, then $A_{k j}=\lambda B_{k j}$ where $B_{k j}$ is an isometry. ${ }^{1}$

Proof. We first prove that the conditions (3.2.1), (3.2.2), (3.2.3), and (3.2.4) are sufficient for $A$ to be adjoint abelian. Let $x=\sum x_{i} \in X$ and $y=\sum y_{i} \in X$ be given. Since there is exactly one nonzero operator per row and column (in the operator matrix) we obtain

$$
[A x, y]=\sum_{k}\left\langle A_{k j} x_{j}, y_{k}\right\rangle \frac{\left\|y_{k}\right\|^{p-2}}{[\nu(y)]^{p-2}}
$$

where $j$ is the unique index corresponding to $k$ guaranteed by (3.2.2). On the other hand,

1 We are indebted to the referee for pointing out that Theorem 3.2 has been proved for the case $X=l^{p}, 1<p<\infty, p \neq 2$, by Twum Owusu Ansah in his doctoral dissertation, Hermitian operators of meromorphic type on Banach spaces, University of Toronto, June, 1972. 


$$
[x, A y]=\sum_{k}\left\langle x_{k}, A_{k j} y_{j}\right\rangle \frac{\left\|A_{k j} y_{j}\right\|^{p-2}}{[\nu(A y)]^{p-2}} .
$$

Since $A_{j k}=A_{k j}^{*}$ we have $\left\langle A_{k j} x_{j}, y_{k}\right\rangle=\left\langle x_{j}, A_{j k} y_{k}\right\rangle$ for each pair $k, j$. Furthermore,

$$
\left[\frac{\left\|A_{j k} y_{k}\right\|}{\nu(A y)}\right]^{p-2}=\left[\frac{\left\|y_{k}\right\|}{\nu(y)}\right]^{p-2}
$$

It follows that the sum (3.2.6) is a rearrangement of the sum (3.2.5) and since each is absolutely convergent, we must have $[A x, y]=[x, A y]$.

Next suppose that $A$ is adjoint abelian on $X$. Let $k, j$ be given positive integers and $x_{k} \in X_{k}, y_{j} \in X_{j}$. Since $A$ is adjoint abelian we must have $\left[A x_{k}, y_{j}\right]=\left[x_{k}, A y_{j}\right]$ and $\left[A y_{j}, x_{k}\right]=\left[y_{j}, A x_{k}\right]$. From these equalities we obtain

$$
\begin{gathered}
\left\langle A_{j k} x_{k}, y_{j}\right\rangle=\left\langle x_{k}, A_{k j} y_{j}\right\rangle \frac{\left\|A_{k j} y_{j}\right\|^{p-2}}{\left[\nu\left(A y_{j}\right)\right]^{p-2}} \text { and } \\
\left\langle A_{k j} y_{j}, x_{k}\right\rangle=\left\langle y_{j}, A_{j_{k}} x_{k}\right\rangle \frac{\left\|A_{j k} x_{k}\right\|^{p-2}}{\left[\nu\left(A x_{k}\right)\right]^{p-2}} .
\end{gathered}
$$

If we take complex conjugates in (3.2.8) and substitute into (3.2.7) we obtain

$$
\left\langle A_{j k} x_{k}, y_{j}\right\rangle=\left\langle A_{j k} x_{k}, y_{j}\right\rangle\left[\frac{\left\|A_{k j} y_{j}\right\|}{\nu\left(A y_{j}\right)}\right]^{p-2}\left[\frac{\left\|A_{j k} x_{k}\right\|}{\nu\left(A x_{k}\right)}\right]^{p-2} .
$$

Suppose there exists $x_{k} \in X_{k}$ and positive integer $n$ such that

$$
A_{n k} x_{k} \neq 0 \text { and } A_{j k} x_{k} \neq 0 .
$$

If there exists $y_{j} \in X_{j}$ such that $\left\langle A_{j_{k}} x_{k}, y_{j}\right\rangle \neq 0$ it follows from (3.2.9) and the fact that $p \neq 2$, that $\left\|A_{k j} y_{j}\right\|=\nu\left(A y_{j}\right)$ and $\left\|A_{j k} x_{k}\right\|=$ $\nu\left(A x_{k}\right)$. Since $\nu\left(A x_{k}\right)=\left(\sum_{m}\left\|A_{m k} x_{k}\right\|^{p}\right)^{1 / p}$, we must have $A_{m k} x_{k}=0$ for all $m \neq j$ which contradicts (3.2.10). Hence $\left\langle A_{j k} x_{k}, y_{j}\right\rangle=0$ for all $y_{j} \in X_{j}$ which implies that $A_{j_{k}} x_{k}=0$, again contradicting (3.2.10). Therefore, if $x_{k} \in X_{k}$, and $n$ is given we must have

(3.2.11) $A_{n k} x_{k}=0$ or $A_{j k} x_{k}=0$. Thus if $A_{j k} \neq 0$, there exists $x_{k} \in X_{k}$ such that $A_{j k} x_{k} \neq 0$. By (3.2.11) $A_{n k} x_{k}=0$. If $A_{n k} \neq 0$, then by (3.2.11) there exists $y_{k} \in X_{k}$ such that $A_{j k} y_{k}=0$. If we let $z_{k}=x_{k}+$ $y_{k}$, then $A_{n k} z_{k}=A_{n k} x_{k}+A_{n k} y_{k}=A_{n k} y_{k} \neq 0$ and $A_{j k} z_{k}=A_{j k} x_{k}+A_{j k} y_{k}=$ $A_{j k} x_{k} \neq 0$. Hence we have $A_{n k} z_{k} \neq 0$ which contradicts (3.2.11). We conclude that

(3.2.12) if $A_{j k} \neq 0$, then $A_{n k}=0$ for all $n \neq j$.

A similar argument would show that if $A_{k j} \neq 0$, then $A_{k n}=0$ for 
all $n \neq j$.

This information, together with (3.2.7) implies that if $A_{j k} \neq 0$, then

$$
\left\langle A_{j k} x_{k}, y_{j}\right\rangle=\left\langle x_{k}, A_{k j} y_{j}\right\rangle \text { for all } x_{k} \in X_{k}, y_{j} \in X_{j} .
$$

Next we show that there exists $\lambda>0$ such that (3.2.14) $\nu(A y)=\lambda \nu(y)$ for all $y \in X$. To see this, let $x=\sum x_{i}, y=\sum y_{i}$ be elements of $x$, then

$$
0=[A x, y]-[x, A y]=\sum_{k}\left\langle A_{k j} x_{j}, y_{k}\right\rangle\left(\frac{\left\|y_{k}\right\|^{p-2}}{[\nu(y)]^{p-2}}-\frac{\left\|A_{j k} y_{k}\right\|^{p-2}}{[\nu(A y)]^{p-2}}\right)
$$

where we have used (3.2.13) and the fact that the sums can be rearranged. By choosing appropriate $x_{j}$ 's, we can show that for each $k$,

$$
\frac{\left\|y_{k}\right\|^{p-2}}{[\nu(y)]^{p-2}}-\frac{\left\|A_{j k} y_{k}\right\|^{p-2}}{[\nu(A y)]^{p-2}}=0 \text { when } A_{k j} \neq 0
$$

Hence for any $k$ such that $y_{k} \neq 0$ we have

$$
\frac{\nu(A y)}{\nu(y)}=\frac{\left\|A_{j k} y_{k}\right\|}{\left\|y_{k}\right\|}
$$

From this we may conclude that $\nu(A y) / \nu(y)$ is constant. To see this let $y, z$ be nonzero elements of $X$. Then there exist integers $k, n$ such that $y_{k}$ and $z_{n}$ are not zero. Let

$$
\lambda=\frac{\nu(A y)}{\nu(y)}=\frac{\left\|A_{j k} y_{k}\right\|}{\left\|y_{k}\right\|} \text { and } \sigma=\frac{\nu(A z)}{\nu(z)}=\frac{\left\|A_{m n} z_{n}\right\|}{\left\|z_{n}\right\|} \text {. }
$$

If $k \neq n$, let $w=y_{k}+z_{n}$. By (3.2.16), we conclude that

$$
\frac{\nu(A w)}{\nu(w)}=\frac{\left\|A_{j_{k}} y_{k}\right\|}{\left\|y_{k}\right\|}=\frac{\left\|A_{m n} z_{n}\right\|}{\left\|z_{n}\right\|}
$$

and hence $\lambda=\sigma$. A similar argument for the case $k=n$ also gives $\lambda=\sigma$. Therefore, $\nu(A y) / \nu(y)=$ constant for all $y \neq 0$. In fact, $\nu(A y) / \nu(y)=\nu_{0}(A)$ whenever $y \neq 0$ and this proves (3.2.14).

Again from (3.2.16) we have

(3.2.17) $\left\|A_{j_{k}} y_{k}\right\|=\lambda\left\|y_{k}\right\|$ for all $y_{k} \in X_{k}$. It is clear from (3.2.14) that every row and column in $\left(A_{k j}\right)$ must have at least one nonzero entry. This fact together with (3.2.12) establishes (3.2.2).

Suppose now that $X_{k}$ and $X_{j}$ have different dimensions and without loss of generality, suppose $\operatorname{dim}\left(X_{k}\right)<\operatorname{dim}\left(X_{j}\right)$. Then $A_{k j}$ cannot be one-to-one as a map from $X_{j}$ to $X_{k}$, which contradicts the fact that $\left\|A_{k j} x_{j}\right\|=\lambda\left\|x_{j}\right\|$ if $A_{k j} \neq 0$. We conclude that $A_{k j}=0$ which establishes (3.2.1). 
If $A_{k j} \neq 0$, then $X_{k}$ and $X_{j}$ are essentially the same space and (3.2.3) follows from (3.2.13).

Finally, if $A_{k j} \neq 0$, then (3.2.17) implies that $\lambda^{-1} A_{k j}$ is an isometry.

REMARK 3.3. The conclusion of Theorem 3.2 holds for operators on a Banach space $X$ as described in $\S 2$ with some additional assumptions on the $a_{k}$ 's which occur in the representation of the s.i.p. as given in 2.5. Those conditions are:

(i ) $a_{k}(x) a_{j}(x)=1$ if and only if $a_{k}(x)=a_{j}(x)=1$.

(ii) $a_{k}(x)=1$ if and only if $a_{j}(x)=0$ for $j \neq k$.

(iii) $a_{k}(x)=a_{j}(y)$ implies $\left\|x_{k}\right\| / \nu(x)=\left\|y_{j}\right\| / \nu(y)$.

REMARK 3.4. Let $X$ be an $l_{p}$ sum of a sequence of Hilbert spaces $\left\{X_{i}\right\}$ where $1<p<\infty$ and $p \neq 2$ and let $H$ be the $l_{2}$ sum of these spaces. If $A$ is an operator which is adjoint abelian on $X$ and if $A$ is defined on $H$ then $A$ is Hermitian on $H$.

REMARK 3.5. If no two $X_{i}$ 's have the same dimension, then an adjoint abelian operator on $X$ has a diagonal matrix representation and therefore is Hermitian by Theorem 2.6.

If, for example, $X$ is the $l_{p}$ sum of a one dimensional and a two dimensional space, then every adjoint abelian operator is Hermitian, but not conversely.

$\operatorname{Thus}\left(\begin{array}{rrr}1 & 0 & 0 \\ 0 & 0 & i \\ 0 & -i & 0\end{array}\right)$ is adjoint abelian on that space while $\left(\begin{array}{rrr}1 & 0 & 0 \\ 0 & 0 & i / 2 \\ 0 & -i / 2 & 0\end{array}\right)$ is Hermitian but not adjoint abelian.

If instead we take $X=X_{1}+X_{2}$ as the $l_{p}$ sum of two 2-dimensional Hilbert spaces where $p \neq 2$, then $\left(\begin{array}{rrrr}0 & 0 & -i & 0 \\ 0 & 0 & 0 & i \\ i & 0 & 0 & 0 \\ 0 & -i & 0 & 0\end{array}\right)$ is an adjoint abelian operator which is not Hermitian. (Here we are considering the matrices as representing an operator relative to the natural coordinate basis vectors.)

It is of some interest perphaps to consider spaces on which the classes of Hermitian and adjoint abelian operators coincide. This happens, of course, on Hilbert spaces. The next theorem shows that this cannot happen on non-Hilbert spaces which are isomorphic to Hilbert spaces. In particular, the two classes are always distinct on finite dimensional spaces.

Let $(X,[\cdot, \cdot])$ be a semi-inner product space. The algebra of bounded operators on $X$ will be denoted by $B(X) . H(X)$ and $A(X)$ will denote the Hermitian and adjoint abelian operators respectively. 
THEOREM 3.6. Let $(X, \nu)$ be a Banach space which is isomorphic to Hilbert space. If there is a s.i.p. $[\cdot, \cdot]$ on $X$, compatible with $\nu$, for which $A(X)=H(X)$ then $(X, \nu)$ is isometric with Hilbert space.

Proof. Suppose $[\cdot, \cdot]$ is a s.i.p. on $X$ compatible with $\nu$ for which $A(X)=H(X)$. Let $T \in H(X)$. Then $T \in A(X)$ and by Lemma 2 of [8] $T^{2} \in H(X)$. By Theorem 3 page 59 of [3], $J=H(X)+i H(X)$ is a Banach* algebra and furthermore it follows from Theorem 5 page 78 of [3] that $J$ is a $B^{*}$ algebra.

Since $X$ is isomorphic to Hilbert space, there is an inner product $\langle\cdot, \cdot\rangle$ defined on $X$ which induces an equivalent norm. Define $S=\{T \in B(X):\langle T x, y\rangle=\langle x, T y\rangle$ for every $x, y\}$. Let $C=S+i S$. Then $C$ is a $B^{*}$ algebra with respect to the involution defined by $\langle T x, y\rangle=\left\langle x, T^{\prime} y\right\rangle$ and $C$ contains the identity. Again by Theorem 5 page 78 of [3] $C \subset J$. But $C=B(X)$. Hence by Theorem 6 page 78 of [3] $X$ is a Hilbert space.

4. Spaces with hyperorthogonal bases and the class $\mathscr{S} \cdot \mathrm{A}$ sequence $\left\{e_{i}\right\}$ in a complex Banach space $(X, \nu)$ is called a Schauder basis if for every $x \in X$ there is a unique sequence $\left\{\alpha_{i}\right\}$ of scalars such that $x=\sum \alpha_{i} e_{i}$. Associated with the sequence $\left\{e_{i}\right\}$ is the sequence $\left\{e_{i}^{*}\right\}$ in the dual space $X^{*}$ defined by $e_{i}^{*}\left(e_{j}\right)=\delta_{i j}$ for each pair $i, j$ of positive integers. Thus $e_{i}^{*}(x)=\alpha_{i}$ and $x=\sum e_{i}^{*}(x) e_{i}$. A basis is sometimes indicated by writing the pair $\left(\left\{e_{i}\right\},\left\{e_{i}^{*}\right\}\right)$. A basis is shrinking if $\left\{e_{i}^{*}\right\}$ is a basis for $X^{*}$ and normal if $\nu\left(e_{i}\right)=\nu^{*}\left(e_{i}^{*}\right)=1$ for each $i$ where $\nu, \nu^{*}$ denote the norm on $X$ and the dual norm on $X^{*}$ respectively. The basis $\left\{e_{i}\right\}$ is said to be hyperorthogonal if $\nu\left(\sum \alpha_{i} e_{i}\right)=$ $\nu\left(\sum\left|\alpha_{i}\right| e_{i}\right)$ for each $x=\sum_{i} \alpha_{i} e_{i} \in X$ [8. p. 558]. If, for $x=\sum \alpha_{i} e_{i}$ we let $|x|=\sum\left|a_{i}\right| e_{i}$, then the above is expressed by saying $\nu(x)=$ $\nu(|x|)$ for each $x \in X$. It may be readily shown that in this case $\nu$ is monotone relative to $\left\{e_{i}\right\}$; that is if $\left|\alpha_{i}\right| \leqq\left|\beta_{i}\right|$ for each $i$ and $\sum \beta_{i} e_{i} \in X$, then $\nu\left(\sum \alpha_{i} e_{i}\right) \leqq \nu\left(\sum \beta_{i} e_{i}\right)$ [8. p. 558].

Let $N$ denote the set of positive integers or the set $\{1,2,3, \cdots, n\}$ for some $n$. We now define an equivalence relation on $N$ in a way suggested by Schneider and Turner [7].

Definition 4.1. If $i, j \in N$ we say $i$ is equivalent to $j$ relative to the norm $\nu$ and the hyperorthogonal basis $\left\{e_{i}\right\}$ for $X$ if $\left|\alpha_{i}\right|^{2}+$ $\left|\alpha_{j}\right|^{2}=\left|\beta_{i}\right|^{2}+\left|\beta_{j}\right|^{2}$ and $\left|\alpha_{k}\right|=\left|\beta_{k}\right|$ for $k \neq i, j$ imply that $\nu\left(\sum \alpha_{k} e_{k}\right)=$ $\nu\left(\sum \beta_{k} e_{k}\right)$. In this case we write $i \sim j$.

It has been shown that " " defines an equivalence relation on $N$ where $N$ is finite [7], and the proof is exactly the same in case $N$ is infinite. We now show that this equivalence relation gives rise to a decomposition of the space in the sense of 2.4 . 
For the remainder of this section, $\left\{e_{i}\right\}$ will denote a normalized (i.e., $\nu\left(e_{i}\right)=1$ for each $i$ ) hyperorthogonal basis for the Banach space $X$ with norm $\nu$. The sets $N_{1}, N_{2}, \cdots$ will denote the equivalence classes in $N$ determined by the equivalence relation of 4.1. We will treat the $N_{i}$ 's as if there are infinitely many of them and each $N_{i}$ is infinite. The necessary adjustments in finite cases are obvious. The elements of $N_{k}$ are denoted by $p_{k 1}, p_{k 2}, \ldots$ in increasing order. If $x=\sum \alpha_{i} e_{i}$ and $\sum\left|\alpha_{i}\right|^{2}<\infty$, we write $\|x\|=\left(\sum\left|\alpha_{i}\right|^{2}\right)^{1 / 2}$.

Lemma 4.2. Let $X, \nu,\left\{e_{i}\right\}, N_{1}, N_{2}, \cdots$ be as described above. Then there exists a sequence of subspaces $\left\{X_{i}\right\}$ of $X$, each being isometric with $l_{2}$, and a sequence space $(E, \mu)$ such that $\left(\left\{X_{i}\right\},(E, \mu)\right)$ is an $H$-decomposition of $X$.

Proof. For each positive integer $i$ let $X_{i}$ denote the closed linear span of the basis vectors $\left\{e_{p_{i k}}: p_{i k} \in N_{i}\right\}$. If $x=\sum \alpha_{k} e_{k} \in X$, let $x_{i}=$ $\sum_{k} \alpha_{p_{i k}} e_{p_{i k}}$. Then $x_{i} \in X_{i}$ and it is readily shown that $x=\sum_{i} x_{i}$. For if $\varepsilon>0$ is given, choose $n_{0}$ such that $\nu\left(\sum_{k \geqq n} \alpha_{k} e_{k}\right)<\varepsilon$ for $n \geqq n_{0}$. Choose $k_{0}$ so large that $\left\{1,2, \cdots, n_{0}\right\} \subset \bigcup_{i<k_{0}} N_{i}$. Then $\nu\left(\sum_{j \geq k} x_{j}\right) \leqq$ $\nu\left(\sum_{j \geqq k} \alpha_{j} e_{j}\right)<\varepsilon$ for $k \geqq k_{0}$. Thus the sequence of subspaces $\left\{x_{i}\right\}$ is a decomposition of $X$.

Let $x^{1}=x-x_{1}$. Since $p_{12} \sim p_{11}$, we have

$$
\nu(x)=\nu\left(x^{1}+\left(\left|\alpha_{p_{11}}\right|^{2}+\left|\alpha_{p_{12}}\right|^{2}\right)^{1 / 2} e_{p_{11}}+\sum \alpha_{p_{1 k}} e_{p_{1 k}}\right) .
$$

By induction it follows that for every $n, k \geqq 3$.

$$
\left.\nu(x)=\dot{\nu\left(x^{1}\right.}+\left(\sum_{k=1}^{n}\left|\alpha_{p_{1 k}}\right|^{2}\right)^{1 / 2} e_{p_{11}}+\sum_{k>n} \alpha_{p_{1 k}} e_{p_{1 k}}\right) .
$$

By the monotone property of $\nu$ we must have

$$
\nu(x) \geqq \nu\left(\left(\sum_{1}^{n}\left|\alpha_{p_{1 k}}\right|^{2}\right)^{1 / 2} e_{p_{11}}\right)=\left(\sum_{1}^{n}\left|\alpha_{p_{1 k}}\right|^{2}\right)^{1 / 2}
$$

for each $n$. We conclude that $\sum_{1}^{\alpha}\left|\alpha_{p_{1 k}}\right|^{2}<\infty$. If $x=x_{1}$, then $\nu(x)=\left\|x_{1}\right\|$ and it is obvious that $X_{1}$ is isometric with $l_{2}$. Since $\nu\left(\sum_{k \geqq n} \alpha_{p_{1 k}} e_{p_{1} k}\right) \rightarrow 0$ as $n \rightarrow \infty$ we may conclude from

(4.2.1) that

$$
\nu(x)=\left(x^{1}+\left\|x_{1}\right\| e_{p_{11}}\right) .
$$

By exactly the same arguments, we may show by induction that if $r$ is a positive integer and $x^{r}=x^{r-1}-x_{r}$,

$$
\nu(x)=\nu\left(x^{r}+\sum_{1}^{r}\left\|x_{k}\right\| e_{p_{k 1}}\right) .
$$


Since

$$
\nu\left(\sum_{k=r}^{r+s}\left\|x_{k}\right\| e_{p_{k 1}}\right)=\nu\left(\sum_{k=r}^{r+s} x_{k}\right), \quad \sum_{1}^{\infty}\left\|x_{k}\right\| e_{p_{k 1}} \in X
$$

and by the monotone property of $\nu$ together with the fact that $\nu\left(x^{r}\right) \rightarrow 0$ we conclude that

$$
\nu(x)=\nu\left(\sum_{1}^{\infty}\left\|x_{i}\right\| e_{p_{i 1}}\right) .
$$

Let $E$ be the space of all scalar sequences $\left\{\alpha_{i}\right\}$ such that $\sum \alpha_{i} e_{p_{i 1}} \in X$ and define $\mu$ on $E$ by $\mu\left(\left\{\alpha_{i}\right\}\right)=\nu\left(\sum \alpha_{i} e_{p_{i}}\right)$. The natural unit vector basis $\left\{u_{i}\right\}$ where $u_{i}=\left(0,0, \cdots, 0,1_{i \mathrm{th}}, 0, \cdots\right)$ is hyperorthogonal and $\mu\left(u_{i}\right)=1$ for each $i$. Moreover, $\left\{\left\|x_{i}\right\|\right\} \in E$ for each $x \in X$ and $\nu(x)=$ $\mu\left(\left\{\left\|x_{i}\right\|\right\}\right)$ by (4.2.4). Therefore, the sequence of subspaces $\left\{X_{i}\right\}$ together with the sequence space $(E, \mu)$ forms an $H$-decomposition of $X$.

COROLlary 4.3. If $i \sim j$ for all $i, j$ then $X$ is isometric with Hilbert space.

REMARK. If the basis for $(X, \nu)$ is also shrinking then it can be shown that $i \sim j$ for $\nu$ implies $i \sim j$ relative to $\nu^{*}$. Furthermore, it follows that $\left(X^{*}, \nu^{*}\right)$ has an $H$-decomposition consisting of the subspaces $\left\{X_{i}^{*}\right\}$. The associated sequence space is a subspace of $\left(E^{*}, \nu^{*}\right)$.

Corollary 4.4. Let $(X, \nu)$ and $\left(\left\{X_{i}\right\},(E, \mu)\right)$ be as in Lemma 4.2. Then no pair of distinct integers $i, j$ can be equivalent relative to $\mu$.

Proof. Suppose there exist positive integers $i, j$ such that $i \sim j$ relative to $\mu$. It is straightforward to verify that if $n \in N_{i}$ and $m \in N_{j}$ then $m \sim n$ relative to $\nu$. But this contradicts the fact that $N_{i} \cap N_{j}=\varnothing$.

We now wish to show that if a Banach space $(X, \nu)$ has an $H$-decomposition $\left(\left\{X_{i}\right\},(E, \mu)\right)$ then the existence of a sufficiently $l_{p}$-like s.i.p. on the sequence space implies that there is a sufficiently $l_{p}$-like s.i.p. on $X$. First we clarify what we mean by a sufficiently $l_{p}$-like semi-inner product on a Banach sequence space $(E, \mu)$ with absolute norm $\mu$.

Definition 4.5. We will call a s.i.p. $[\cdot, \cdot]$ on $(E, \mu)$ sufficiently $l_{p}$-like if there exists a sequence of nonnegative real-valued functions $\left\{a_{i}\right\}$ defined on $X$ such that:

(4.5.1) $a_{k}(\alpha)=a_{k}(\beta)$ for each $k$ if $\left|\alpha_{i}\right|=\left|\beta_{i}\right|$ for each $i$, 
(4.5.2) $a_{k}(\lambda \alpha)=a_{k}(\alpha)$ for every scalar $\lambda \neq 0$ and positive integer $k$,

(4.5.3) For every pair of positive integers $k \neq j$ there exist scalars $\alpha_{k}, \alpha_{j}$ such that $a_{k}(\alpha)=a_{j}(\alpha) \neq 0$ whenever

$$
\alpha=\left(0,0 \cdots \stackrel{(k)}{\alpha_{k}}, 0.0 ., \stackrel{(j)}{\alpha}, 0 \cdots\right),
$$

(4.5.4) For every pair of positive integers $k \neq j$, there exists $\alpha \in E$ with $\alpha_{k} \neq 0, \alpha_{j} \neq 0$ such that $a_{k}(\alpha) \neq \alpha_{j}(\alpha)$,

$$
[\alpha, \beta]=\sum \alpha_{i} \bar{\beta}_{i} a_{i}(\beta) \text {. }
$$

REMARK. An example of a Banach sequence space with absolute norm which possesses a sufficiently $l_{p}$-like s.i.p. is $l_{p} 1 \leqq p<\infty(p \neq 2)$. In this case $[\alpha, \beta]=\sum \alpha_{i} \bar{\beta}_{i}\left(\frac{\left|\beta_{i}\right|}{\|\beta\|_{p}}\right)^{p-2}$.

Lemma 4.6. Let $(X, \nu)$ be a Banach space with an H-decomposition $\left(\left\{X_{i}\right\},(E, \mu)\right) . \quad$ If $(E, \mu)$ possesses a sufficiently $l_{p}$-like semi-inner product then $(X, \nu)$ possesses a sufficiently $l_{p}$-like semi-inner product.

Proof. Given $\alpha=\left\{\alpha_{i}\right\}, \beta=\left\{\beta_{i}\right\} \in E$, let $[\alpha, \beta]=\sum \alpha_{i} \bar{\beta}_{i} a_{i}(\beta)$ represent a sufficiently $l_{p}$-like s.i.p. on $E$. We first show that the s.i.p. on $E$ yields a s.i.p. on $X$. Let $x=\sum x_{i}, y=\sum y_{i}$ be elements of $X$, then $\alpha=\left\{\left\|x_{\imath}\right\|\right\}, \beta=\left\{\left\|y_{i}\right\|\right\} \in E$. We define $[x, y]=\sum\left\langle x_{i}, y_{i}\right\rangle A_{i}(y)$ where $A_{2}(y)=a_{i}(\beta)$. It is obvious that $[\cdot, \cdot]$ is linear in the first argument and $[x, x]=\nu^{2}(x)$. Furthermore,

$$
\begin{aligned}
|[x, y]|^{2} & =\left|\sum\left\langle x_{i}, y_{i}\right\rangle A_{i}(y)\right|^{2} \leqq\left(\sum\left\|x_{i}\right\|\left\|y_{i}\right\| a_{i}(\beta)\right)^{2} \\
& =|[\alpha, \beta]|^{2} \leqq \mu(\alpha) \mu(\beta)=\nu(x) \nu(y) .
\end{aligned}
$$

Thus, $[\cdot, \cdot]$ is a s.i.p. on $X$ compatible with $\nu$. We now proceed to show that the s.i.p. is sufficiently $1_{p}$-like in the sense of 2.5.

It is obvious that (2.5.1), (2.5.2), (2.5.5) hold. To see that (2.5.3) holds, let $k \neq j$ be given. There exists a sequence $\alpha=\left(0,0, \cdots, \alpha_{k}, 0, \cdots\right.$, $\left.\alpha_{j}, 0,0\right) \in E$ such that $a_{k}(\alpha)=a_{j}(\alpha)$. Let $x_{k}, x_{j}$ be nonzero elements of $X_{k}$ and $X_{j}$ respectively. Define $z=\alpha_{k}\left(x_{k} /\left\|x_{k}\right\|\right)+\alpha_{j}\left(x_{j} /\left\|x_{j}\right\|\right)$. Then $A_{k}(z)=a_{k}(\alpha)=A_{j}(z)$. A similar argument proves (2.5.4) and this completes the proof of the lemma.

TheOREM 4.7. Let $(X, \nu)$ be a Banach space with a normalized hyperorthogonal basis. Then $X$ has a sufficiently $l_{p}$-like semi-inner product which is compatible with the norm.

Proof. Since $X$ has a hyperorthogonal basis, it follows from Lemma 4.2 that $X$ possesses an $H$-decomposition $\left(\left\{X_{i}\right\},(E, \mu)\right)$. By Lemma 4.6 it is sufficient to prove that the sequence space $(E, \mu)$ 
has a sufficiently $l_{p}$-like s.i.p. compatible with $\mu$.

In what follows, the dual of $E$ will be denoted by $E^{*}$ and the associated norm will be denoted by $\mu^{*}$. Since $\mu$ is absolute, $\mu^{*}$ is also absolute.

Let $\phi$ be a duality map $\phi: E \rightarrow E^{*}$ with the properties

$$
\mu^{*}(\phi(\alpha))=\mu(\alpha), \phi(\alpha)(\alpha)=\mu^{2}(\alpha) \text {, and } \phi(\lambda \alpha)=\bar{\lambda} \phi(\alpha) \text {. }
$$

A semi-inner product compatible with $\mu$ is given by

(4.7.1) $[\alpha, \beta]=\phi(\beta)(\alpha)$. We proceed to show that this s.i.p. is sufficiently $l_{p}$-like in the sense of Definition 4.5.

Since $E$ has a basis we can associate with the linear functional $\phi(\alpha)$ a sequence of scalars $\left\{\phi_{i}(\alpha)\right\}$ such that for any $\beta \in E, \phi(\alpha)(\beta)=$ $\sum \beta_{i} \phi_{i}(\alpha)$. From the absoluteness of the norms $\mu$ and $\mu^{*}$ we have $\mu^{*}\left(\left\{\left|\phi_{i}(\alpha)\right|\right\}\right)=\mu^{*}\left(\left\{\phi_{i}(\alpha)\right\}\right)=\mu(\alpha)$. It follows that

$$
\begin{aligned}
\mu^{2}(\alpha) & =\phi(\alpha)(\alpha)=\sum \alpha_{i} \dot{\phi}_{i}(\alpha) \leqq \sum\left|\phi_{i}(\alpha)\right|\left|\alpha_{i}\right| \\
& \leqq \mu^{*}\left(\left\{\left|\phi_{\imath}(\alpha)\right|\right\}\right) \mu\left(\left\{\left|\alpha_{i}\right|\right\}\right)=\mu^{2}(\alpha) .
\end{aligned}
$$

From (4.7.2) we may conclude that $\phi_{i}(\alpha) \alpha_{i}=\left|\phi_{i}(\alpha)\right|\left|\alpha_{i}\right|$ and consequently $\phi_{i}(\alpha) / \bar{\alpha}_{i}$ is real for every $i$ such that $\alpha_{i} \neq 0$. If we define,

$$
a_{i}(\alpha)=\left\{\begin{array}{ccc}
\frac{\phi_{i}(\alpha)}{\bar{\alpha}_{i}} & \text { if } \quad \alpha_{i} \neq 0 \\
0 & \text { if } \quad \alpha_{i}=0 \text { then, }
\end{array}\right.
$$

(4.7.4) $[\alpha, \beta]=\sum \alpha_{i} \phi_{i}(\beta)=\sum \alpha_{i} \bar{\beta}_{i} a_{i}(\beta)$ for any choice of $\alpha, \beta \in E$. To show that this s.i.p. is sufficiently $1_{p}$-like we must now verify that the $a_{\imath}$ 's satisfy the conditions of Definition 4.5.

It is obvious that (4.5.1), (4.5.2), and (4.5.5) hold. It follows from Corollary 3.2 of [7] that given a pair of positive integers $k, j$ there exist positive scalars $\alpha_{k}, \alpha_{j}$ such that $a_{k}(\alpha)=a_{j}(\alpha)$ if $\alpha=\left(0,0, \alpha_{k}, 0, \alpha_{j}\right.$, $0, \cdots)$. Thus, (4.5.3) is satisfied.

To show that (4.5.4) holds, let $k, j, k \neq j$ be given and suppose $a_{k}(\alpha)=a_{j}(\alpha)$ for all $\alpha \in E$. Consider the subspace $E_{n}$ of $E$ spanned by $\left\{u_{1}, u_{2}, \cdots, u_{n}\right\}, n \geqq \max \{k, j\}$. A s.i.p. on $E_{n}$ compatible with $\mu_{n}$, the norm $\mu$ restricted to $E_{n}$, is given by $[\alpha, \beta]_{n}=\sum \alpha_{i} \bar{\beta}_{i} \alpha_{i}(\beta)$. Let $B$ be the matrix $\left(b_{p q}\right)$ defined by $b_{k j}=i, b_{j k}=-i$ and $b_{p q}=0$ for all other choices of $p, q$. It is readily verified that $B$ is Hermitian and by the theorem of Schneider and Turner [7, Theorem 6.2] $k \sim j$ relative to $\mu_{n}$. A limiting argument shows that $k \sim j$ relative to $\mu$ which contradicts Corollary 4.4. Hence there must exist $\alpha \in E$ such that $a_{k}(\alpha) \neq a_{j}(\alpha)$ and the proof is complete.

As a consequence of Theorem 4.7 we know that any Banach 
space $(X, \nu)$ which has a normalized hyperorthogonal basis is a member of the class $\mathscr{S}$ discussed in $\S 2$. These results as applied to a Banach space with a normalized hyperorthogonal basis are summarized in the next theorem.

THEOREM 4.8. Let $(X, \nu)$ be a Banach space with a normalized hyperorthogonal basis. Then

(i) there exists a sequence of subspaces $\left\{X_{i}\right\}$, each a Hilbert space, which decompose $X$.

(ii) every linear operator $A$ on $X$ may be represented by an operator matrix $\left(A_{i j}\right)$ where $A_{i j}: X_{j} \rightarrow X_{i}$.

(iii) an operator $A=\left(A_{i j}\right)$ on $X$ is Hermitian iff $A_{k k}$ is Hermitian on $X_{k}$ for each $k, A_{k j}=0$ for $k \neq j$. Furthermore, the norm of $A$ is $\sup _{k}\left\|A_{k k}\right\|$.

(iv) the product of two Hermitian operators is Hermitian if and only if the operators commute.

(v) every power $A^{n}$ of a Hermitian operator $A$ is Hermitian.

(vi) an operator $A=\left(A_{k j}\right)$ is normal if and only if $A_{k j}=0$ for $k \neq j$ and $A_{k k}$ is a normal operator on the Hilbert space $X_{k}$.

REMARKs. If $A$ is regarded as an infinite matrix $\left(a_{i j}\right)$ of scalars, and $A$ is Hermitian on $X$, then

(i) $a_{i j}=\bar{a}_{j i}$ for each $i, j$.

(ii) $a_{i j}=0$ if $i \neq j$.

This extends the theorem of Schneider and Turner [7]. Also, our results on Hermitian and normal operators are valid in any discrete Banach function space with absolutely continuous norm and therefore extend the results of Tam in that we do not require symmetry of the norm.

Acknowledgement. We wish to express our appreciation to David Saunders of the University of Wisconsin for pointing out a difficulty in the proof of Theorem 2.6 in an earlier version of the paper. We also wish to thank the referee for several helpful comments and suggestions.

\section{REFERENCES}

1. F. L. Bauer, On the field of values subordinate to a norm, Numer. Math., 4 (1962), 103-111.

2. E. Berkson, Hermitian projections and orthogonality in Banach spaces, Proc. London Math. Soc., (3) 24 (1972), 101-118.

3. F. F. Bonsall and J. Duncan, Numerical ranges of operators in normed spaces and of elements of normed algebras, London Math. Soc. Lecture Note Series (2), 1971.

4. G. Lumer, Semi-inner product spaces, Trans. Amer. Math. Soc., 100 (1961), 29-43.

5. On the isometries of reflexive Orlicz spaces, An. Inst. Fourier; Grenoble, 
13 (1963), 99-109.

6. G. Lumer, Spectral operators, Hermitian operators and bounded groups, Acta. Sci. Math. (Szeged), 25 (1964), 75-85.

7. H. Schneider and R. E. L. Turner, Matrices Hermitian for an absolute norm, to appear.

8. I. Singer, Bases in Banach Spaces I, Springer-Verlag, Berlin, 1970.

9. J. G. Stampfli, Adjoint abelian operators on Banach space, Canad. J. Math., 21 (1969), 505-512.

10. K. W. Tam, Isometries of certain function spaces, Pacific J. Math., 31 (1969), 233-246.

Received February 9, 1973 and in revised form June 14, 1973.

Memphis State University 


\section{PACIFIC JOURNAL OF MATHEMATICS}

EDITORS

RICHARD ARENS (Managing Editor)

University of California

Los Angeles, California 90024

R. A. Beaumont

University of Washington

Seattle, Washington 98105

J. DugundJI

Department of Mathematics

University of Southern California

Los Angeles, California 90007

D. Gilbarg and J. Milgram

Stanford University

Stanford, California 94305

\section{ASSOCIATE EDITORS}

E. F. BECKENBACH

B. H. NeumanN

F. WOLF

K. YOSHIDA

\section{SUPPORTING INSTITUTIONS}

UNIVERSITY OF BRITISH COLUMBIA CALIFORNIA INSTITUTE OF TECHNOLOGY

UNIVERSITY OF CALIFORNIA

MONTANA STATE UNIVERSITY

UNIVERSITY OF NEVADA

NEW MEXICO STATE UNIVERSITY

OREGON STATE UNIVERSITY

UNIVERSITY OF OREGON

OSAKA UNIVERSITY

\author{
UNIVERSITY OF SOUTHERN CALIFORNIA \\ STANFORD UNIVERSITY \\ UNIVERSITY OF TOKYO \\ UNIVERSITY OF UTAH \\ WASHINGTON STATE UNIVERSITY \\ UNIVERSITY OF WASHINGTON \\ $\stackrel{*}{*} \stackrel{*}{*}{ }^{*}{ }^{*}{ }^{2}$ AMERICAN MATHEMATICAL SOCIETY \\ NAVAL WEAPONS CENTER
}




\section{Pacific Journal of Mathematics}

\section{Vol. 52, No. $1 \quad$ January, 1974}

David R. Adams, On the exceptional sets for spaces of potentials ............ 1

Philip Bacon, Axioms for the Čech cohomology of paracompacta ............ 7

Selwyn Ross Caradus, Perturbation theory for generalized Fredholm operators ..... 11

Kuang-Ho Chen, Phragmén-Lindelöf type theorems for a system of nonhomogeneous equations ............................ 17

Frederick Knowles Dashiell, Jr., Isomorphism problems for the Baire classes .......

M. G. Deshpande and V. K. Deshpande, Rings whose proper homomorphic images are right subdirectly irreducible . . . . . . . . . . . . . . . . . . . . . . . . .

Mary Rodriguez Embry, Self adjoint strictly cyclic operator algebras .............

Paul Erdős, On the distribution of numbers of the form $\sigma(n) / n$ and on some related

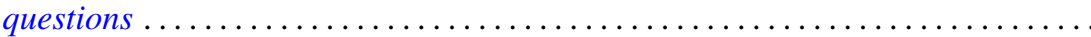

Richard Joseph Fleming and James E. Jamison, Hermitian and adjoint abelian

operators on certain Banach spaces ............................

Stanley P. Gudder and L. Haskins, The center of a poset .................. 85

Richard Howard Herman, Automorphism groups of operator algebras . . . ........

Worthen N. Hunsacker and Somashekhar Amrith Naimpally, Local compactness of families of continuous point-compact relations ....................

Donald Gordon James, On the normal subgroups of integral orthogonal groups ....

Eugene Carlyle Johnsen and Thomas Frederick Storer, Combinatorial structures in

loops. II. Commutative inverse property cyclic neofields of prime-power

order.

Ka-Sing Lau, Extreme operators on Choquet simplexes . . . . . . . . . . . . . . 129

Philip A. Leonard and Kenneth S. Williams, The septic character of 2, 3, 5 and $7 \ldots 143$

Dennis McGavran and Jingyal Pak, On the Nielsen number of a fiber map ........ 149

Stuart Edward Mills, Normed Köthe spaces as intermediate spaces of $L_{1}$ and

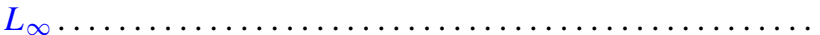

Philip Olin, Free products and elementary equivalence. .

Louis Jackson Ratliff, Jr., Locally quasi-unmixed Noetherian rings and ideals of the principal class.

Seiya Sasao, Homotopy types of spherical fibre spaces over spheres ...

Helga Schirmer, Fixed point sets of polyhedra ...

Kevin James Sharpe, Compatible topologies and continuous irreducible

representations.

Frank Siwiec, On defining a space by a weak base . . . . . . . . . . . . . . . 233

James McLean Sloss, Global reflection for a class of simple closed curves ....... 247

M. V. Subba Rao, On two congruences for primality . .

Raymond D. Terry, Oscillatory properties of a delay differential equation of even

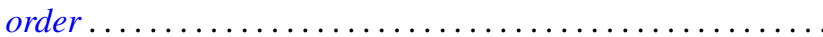

Joseph Dinneen Ward, Chebyshev centers in spaces of continuous functions . .

Robert Breckenridge Warfield, Jr., The uniqueness of elongations of Abelian

groups...

V. M. Warfield, Existence and adjoint theorems for linear stochastic differential

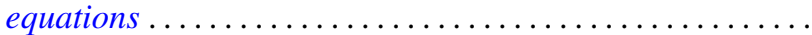

